\title{
ENGENHARIA DE SISTEMAS DE SISTEMAS APLICADA À PROSPECÇÃO DOS REQUISITOS LOGÍSTICOS E INDUSTRIAIS DO SISTEMA DE ARMAS MA- NUAL PARA AS VIATURAS DA FAMÍLIA GUARANI
}

\author{
Rubem Cruz de Aragão \\ Diretoria de Fabricação \\ Av Presidente Vargas, 25, $7^{\circ}$ andar - Centro/RJ \\ aragao.rubem@eb.mil.br \\ Danielle Souza Bonifácio \\ Diretoria de Fabricação \\ Av Presidente Vargas, 25, $7^{\circ}$ andar - Centro/RJ \\ danibonifacio@gmail.com \\ Cicero R Garcez \\ Instituto Militar de Engenharia \\ Praça Gen Tibúrcio, 80 - Urca/RJ \\ garcez@ime.eb.br
}

\section{RESUMO}

Requisitos Logísticos e Industriais são parte do conjunto de requisitos necessários para a obtenção de qualquer produto. Sua abordagem por meio da Engenharia de Sistemas permite aos usuários uma visão sistêmica, sinérgica e empática do programa em que o sistema em estudo está inserido. Neste trabalho, foram realizados estudos de prospecção dos requisitos Logísticos e Industriais de um Sistema de Armas Manual (SAM), sistema esse integrante da Nova Família de Blindados Sob Rodas GUARANI (NFBR GUARANI), mediante o uso de Diagramas SysML/UML e INCOSE.

Palavra-chave: Requisitos Logísticos 1; Requisitos Industriais 2; Engenharia de Sistemas 3; Sistema de Armas Manual 4; Guarani 5.

\begin{abstract}
Logistic and Industrial Requirements are part of the set of requirements required to obtain any product. Its approach through Systems Engineering allows users a systemic, synergistic and empathic view of the program in which the system under study is inserted. In this work, studies were carried out to prospect the Logistic and Industrial requirements of a Manual Weapons System (SAM), a system that is part of the New GUARANI Wheeled Armor Family (NFBR GUARANI), using SysML / UML and INCOSE Diagrams.
\end{abstract}

Keywords: Logistic Requirements 1; Industrial Requirements 2; Systems Engineering 3; Manual Weapon System 4; Guarani 5. 


\section{INTRODUÇÃO}

O presente trabalho tem por objetivo a Prospecção dos Requisitos Logísticos e Industriais (RLI) do Sistema de Armas Manual (SAM) do Programa Estratégico do Exército GUARANI para fabricação no Arsenal de Guerra do Rio (AGR), sob a ótica da Engenharia de Sistema de Sistemas (ESS).

Para a elaboração deste trabalho foi necessário realizar uma análise do contexto do Programa Guarani, buscando dados e informações relevantes sobre os requisitos dos stakeholders, sua análise, bem como a dos requisitos operacionais do SAM e os consequentes requisitos logísticos e industriais.

Na sequência, foi feita uma revisão da metodologia da ESS com o intuito de familiarizar-se com suas ferramentas e identificar que tipos de diagramas poderiam ser aderentes com as demandas do Programa Guarani, de modo geral e ao SAM, de modo específico.

No decorrer dos estudos em comento, foram percebidas o quão valioso é a ESS no sentido de revelar muitas lacunas do Programa Guarani e que poderiam ser evitadas se sua metodologia tivesse sido aplicada desde o início.

É bastante encorajador para a ESS ter sua metodologia utilizada pela maior potência militar da atualidade. Os Estados Unidos da América (EUA), por meio do seu Departamento de Defesa (Department of Defense - DoD), elaborou o Defense Acquisition Guidebook (DAG), em que delineia todo o processo de aquisições de Produtos de Defesa (PRODE) Norte-americanos, utilizado a Engenharia de Sistemas como ferramenta base, dedicando o Capítulo 3 inteiramente a ele.

Apenas como exemplo da importância dada à ESS, todo o programa de desenvolvimento de algum PRODE deve obedecer ao preconizado no DAG e, particularmente, elaborar o System Engineering Plan (SEP), ou Plano de Engenharia de Sistema. Esse documento obriga o Gerente do Programa a conduzir todas as fases do Programa de acordo com os princípios e as ferramentas da ESS.

Essa referência permitiu identificar diversas lacunas no Programa Guarani que serão expostas no decorrer deste trabalho.

\section{DIAGRAMAS APLICADOS AOS REQUISITOS LOGÍSTICOS E INDUSTRIAIS}

O desenvolvimento deste trabalho foi feito em duas etapas: criação dos diagramas de contexto e criação dos diagramas domínio.

Os diagramas de contexto usados neste trabalho são: SIMILAR, EAP, Diagrama de blocos e GANTT. E os diagramas de domínio utilizados foram os diagramas de requisitos para os requisitos logísticos e para os requisitos industriais.

\subsection{DIAGRAMAS DE CONTEXTO}

O Diagrama SIMILAR, promovido pelo INCOSE mostra as etapas de apresentação do problema, investigação de alternativas, modelagem do sistema, integração, iniciação do sistema, avaliação do desempenho e reavaliação ligada à todas as etapas.

A etapa de apresentação do problema foi definida pela da Demanda da Diretoria de Fabricação como sendo a necessidade de se obter os requisitos logísticos industriais para um Sistema de Armas Manual para as viaturas desenvolvidas.

A investigação das alternativas foi dividida em duas partes: forma de obtenção e modelo de proteção balística. A forma de obtenção baseou-se nas escolhas dentre as opções de obtenção do Sistemas de Armas, a saber: nacionalização, aquisição e fabricação. Para este 
estudo, optou-se- pela forma de obtenção como sendo a fabricação pelo AGR já que esta é a opção de maior valor agregado para os estudos de requisitos logísticos industriais. As alternativas para o modelo de proteção foram: proteção frontal, proteção frontal e lateral e proteção frontal, lateral e superior. Optou-se pela proteção completa, qual seja, proteção frontal, lateral e superior.

A modelagem do sistema consistiu em se obter os desdobramentos dos requisitos. A EAP e os diagramas de domínios foram os resultados desta etapa.

As demais etapas do diagrama só se permitem a realização após execução a materialização do objeto estudado. A proposta de fabricação pelo AGR é apenas uma expectativa que não será viabilizada no corrente ano. Sendo assim, as etapas de integração e implementação ficam prejudicadas já que não se possui o objeto de estudo de forma concreta.

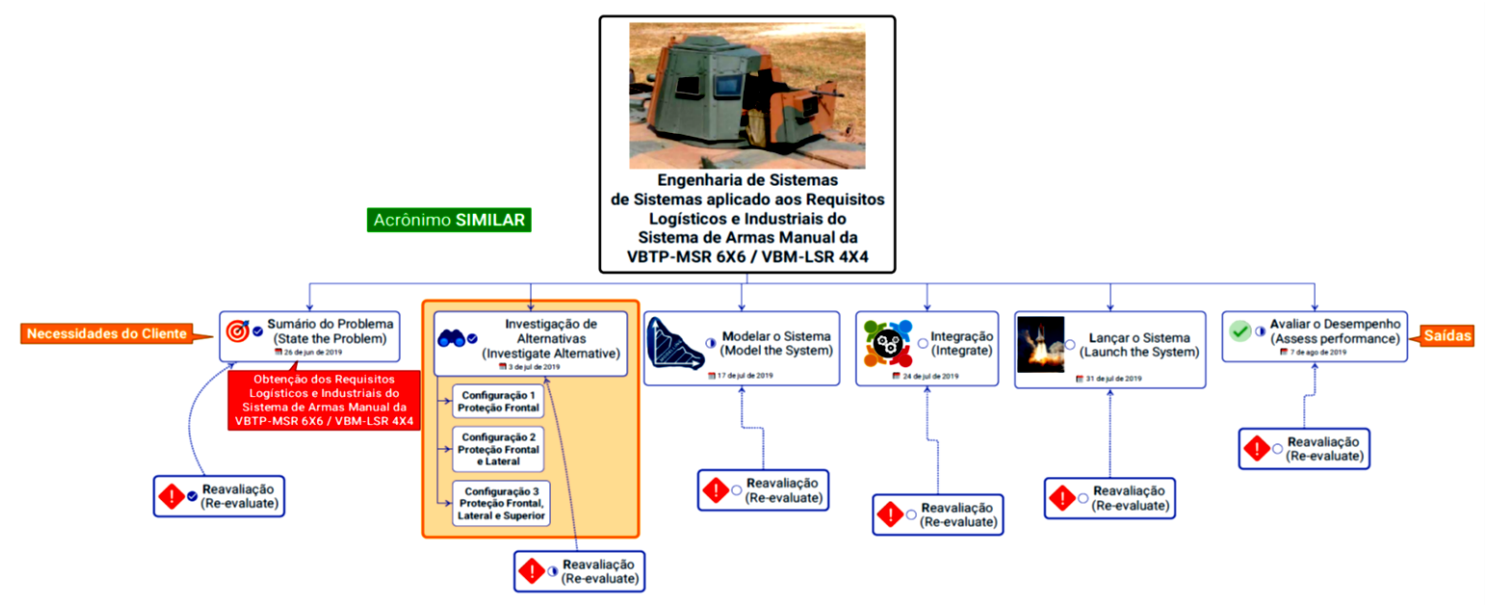

\section{Diagrama SIMILAR}

A EAP do trabalho mostra os grandes grupos de atividades e conhecimentos a serem inseridos na definição dos requisitos logísticos e industriais, de forma interligada e hierárquica dentro de cada categoria. A figura abaixo mostra como ficou a EAP para este trabalho. 


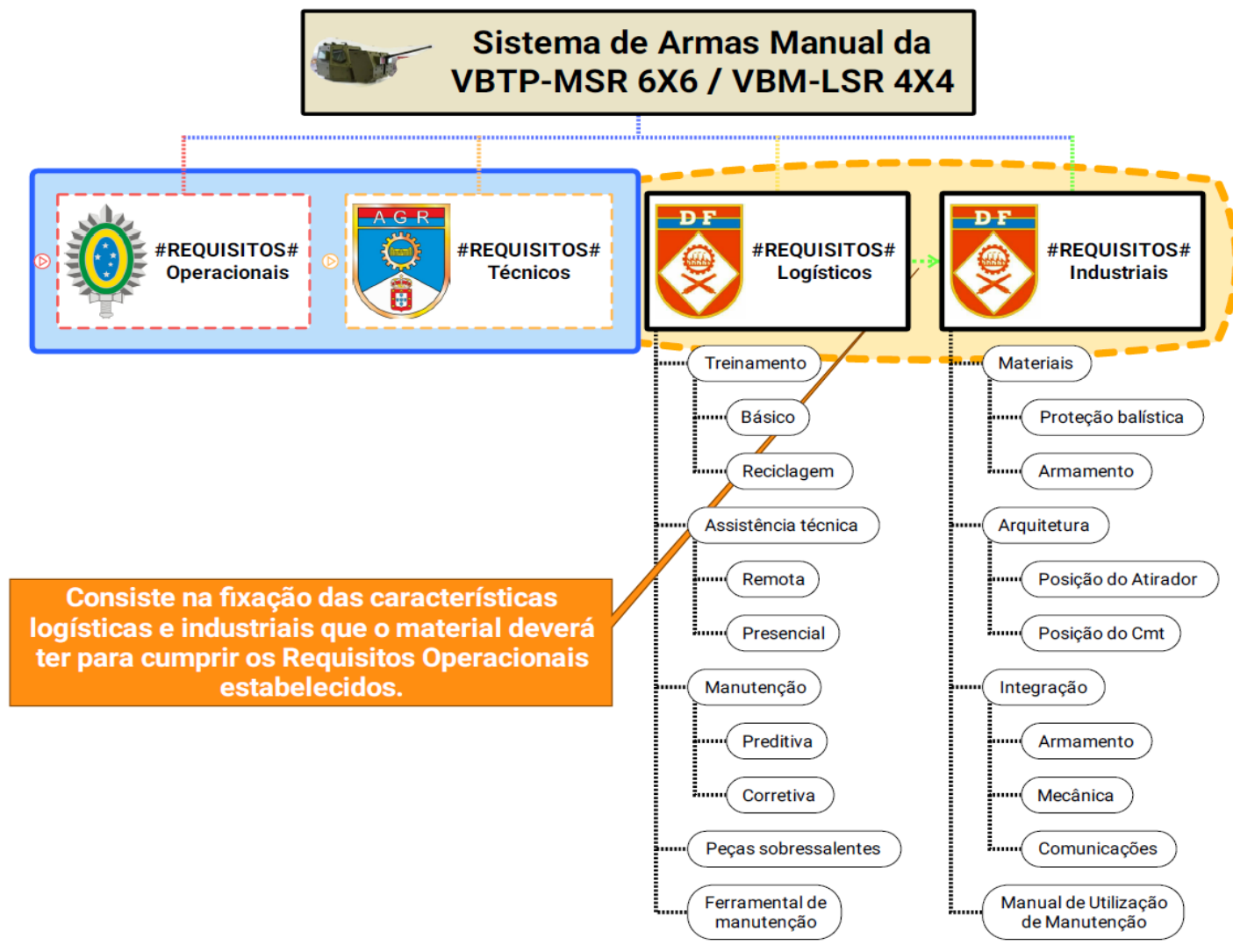

EAP para os Requisitos Logísticos e Industriais

O modelo de diagrama de blocos utilizado mostra as ligações do objeto de estudo com os stakeholders e ainda como funciona o fluxo de informações e quais as entregas entre eles. A figura abaixo mostra o objeto de estudo - Sistemas de Armas Manual - no centro do diagrama. Ele recebe do Escritórios de Projetos do Exército (EPEx) a definição dos requisitos operacionais e Técnicos já elencando. O Arsenal de Guerra do Rio (AGR) incorpora dados e informações ao projeto e a Diretoria de Fabricação realiza a orientação do trabalho. A saída (ou entrega) desse sistema são exatamente os Requisitos Logísticos Industriais para fabricação do Sistema de Armas Manual pelo AGR.

O diagrama de GANTT utilizado contribui para o acompanhamento da execução do trabalho e a divisão de tarefas do grupo de estudo. Cada etapa é demarcada por período de tempo e responsável, assim é possível acompanhar a progressividade de andamento de cada etapa. A mostra o diagrama na etapa intermediária do trabalho. 


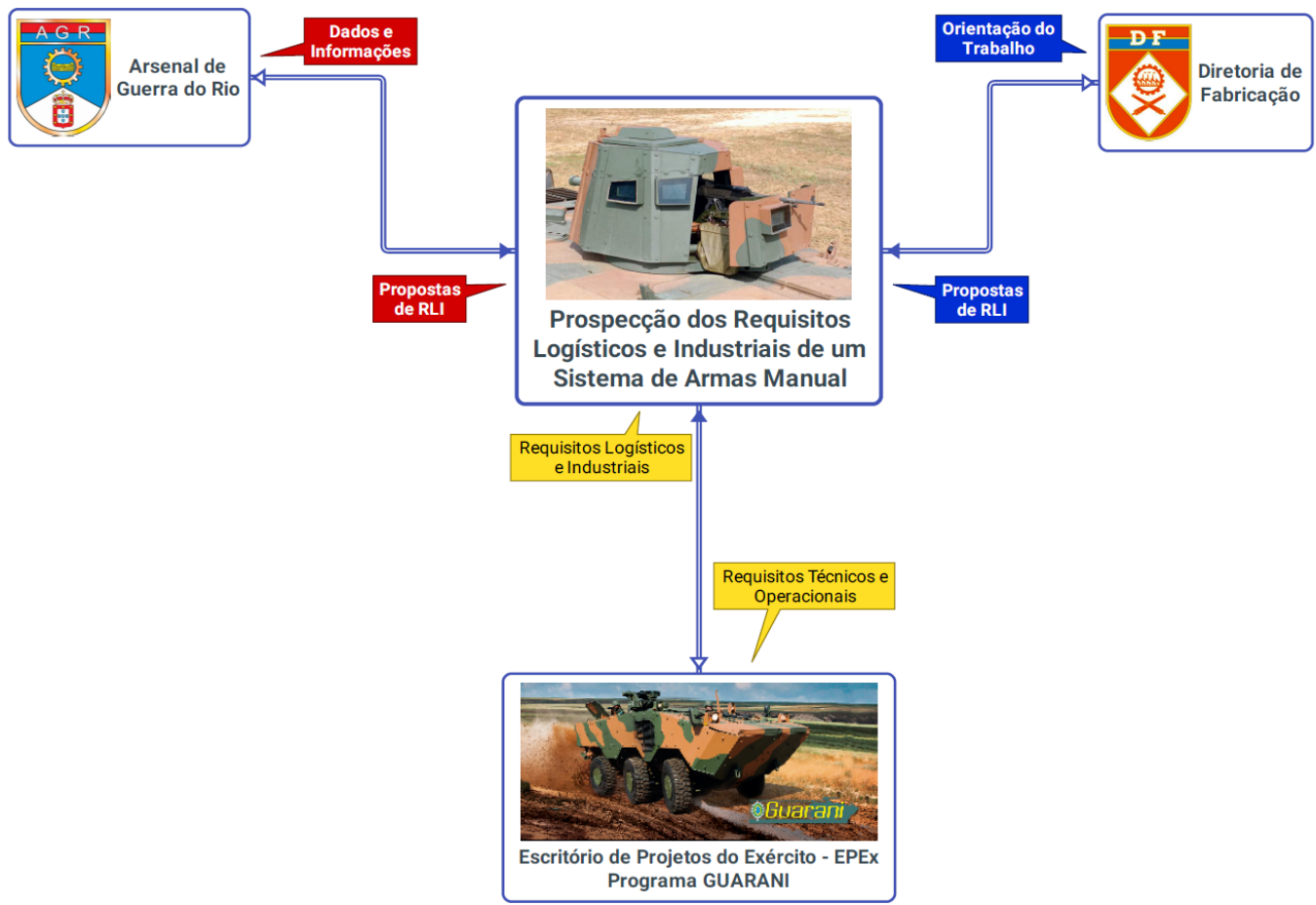

Diagrama de Blocos

O diagrama de GANTT utilizado contribui para o acompanhamento da execução do trabalho e a divisão de tarefas do grupo de estudo. Cada etapa é demarcada por período de tempo e responsável, assim é possível acompanhar a progressividade de andamento de cada etapa. A figura abaixo mostra o diagrama na etapa intermediária do trabalho.

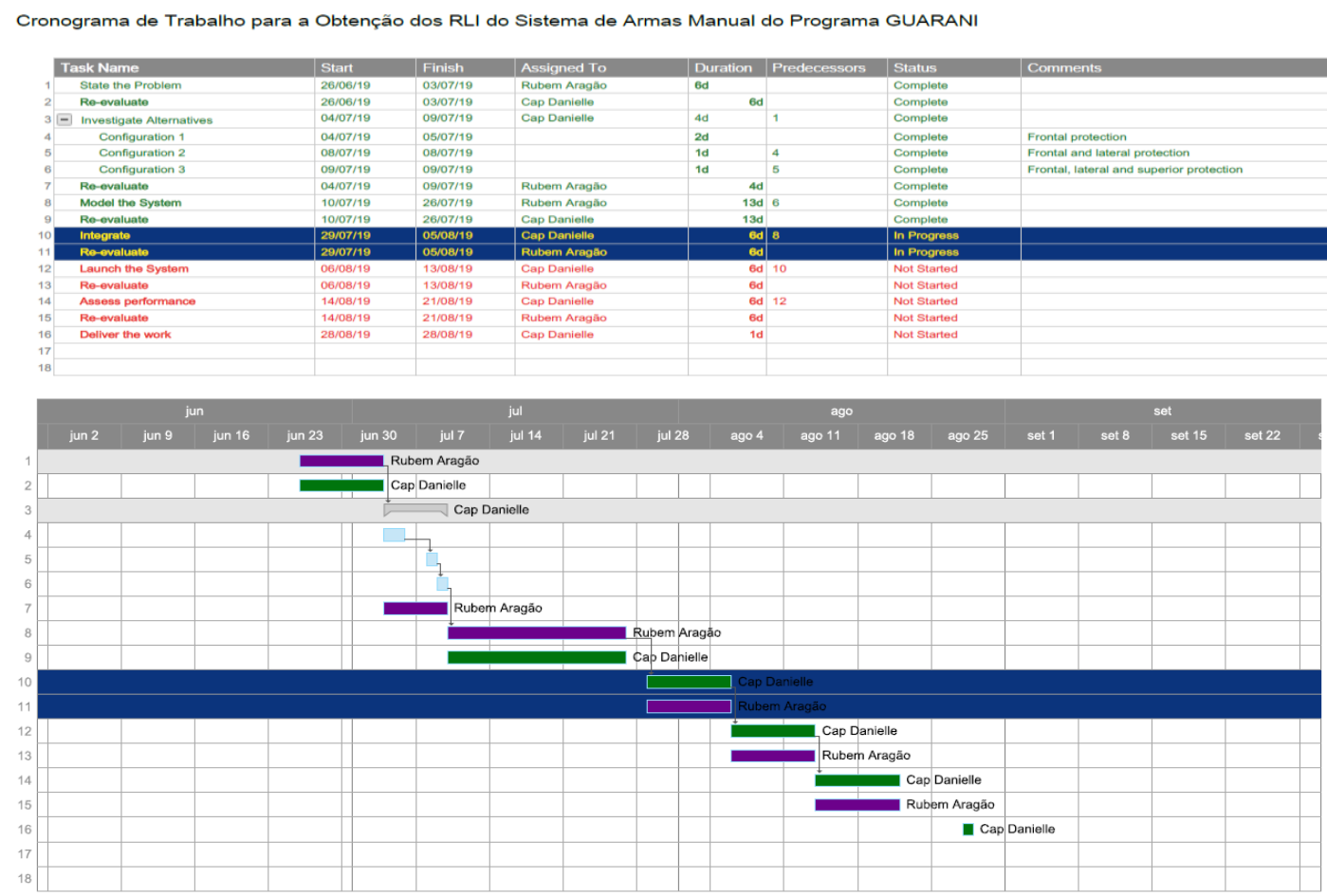

Diagrama de GANTT 
O Diagrama de GANTT foi dividido em duas partes devido à sua extensão horizontal. Esta extensão é o retrato do calendário de execução da atividade.

\subsection{DIAGRAMAS DE DOMÍNIO}

Os diagramas de domínio utilizados foram os Diagramas de Requisitos do SysML, na sua forma padrão e na sua forma tabular.

Há uma sequência de confecção de requisitos dada pela seguinte ordem: operacionais, técnicos, logísticos e industriais. Não há uma hierarquia entre eles, apenas uma ordem de surgimento, já que os requisitos técnicos, logísticos e industriais advêm dos requisitos operacionais.

Para o Sistema de Armas Manual, os requisitos operacionais foram definidos no rol de requisitos operacionais da viatura. A Tabela Erro! Fonte de referência não encontrada. mostra os requisitos operacionais do Sistema de Armas Manual.

Tabela Erro! Fonte de referência não encontrada.

\begin{tabular}{|c|c|}
\hline ID & REQUISITO OPERACIONAL \\
\hline $\begin{array}{l}\text { ROA } \\
46-1\end{array}$ & \multirow[b]{2}{*}{$\begin{array}{l}\text { Possuir torre com movimento horizontal com acionamento } \\
\text { assistido de } \mathrm{n} \times 360^{\circ} \text { (trezentos e sessenta graus), guarnecida por } \\
\text { dois homens (comandante e atirador), com mecanismo de } \\
\text { segurança em caso de falha do mesmo. }\end{array}$} \\
\hline $\begin{array}{l}\text { ROA } \\
46-2\end{array}$ & \\
\hline $\begin{array}{l}\text { ROA } \\
49\end{array}$ & $\begin{array}{l}\text { Possuir na torre, como armamento secundário, uma metralhadora } \\
7,62 \times 51 \mathrm{~mm} \text { (sete vírgula sessenta e dois por cinquenta e um } \\
\text { milímetros) coaxial. }\end{array}$ \\
\hline $\begin{array}{l}\text { ROD } \\
7\end{array}$ & $\begin{array}{l}\text { Possuir na torre, como armamento secundário, uma metralhadora } \\
7,62 \times 51 \mathrm{~mm} \text { (sete vírgula sessenta e dois por cinquenta e um } \\
\text { milímetros) para o comandante do carro. }\end{array}$ \\
\hline $\begin{array}{l}\text { ROD } \\
15-1\end{array}$ & \multirow{2}{*}{$\begin{array}{l}\text { Possuir, na torre, periscópios de visão diurna para o comandante } \\
\text { da viatura e para o atirador. }\end{array}$} \\
\hline $\begin{array}{l}\text { ROD } \\
15-2\end{array}$ & \\
\hline
\end{tabular}

Destes requisitos operacionais foram obtidos a listagem geral de requisitos logísticos e requisitos industriais. A TabelaErro! Fonte de referência não encontrada. mostra o elenco de requisitos e o detalhamento de cada grupo. 
Tabela Erro! Fonte de referência não encontrada.

\begin{tabular}{|c|c|c|c|}
\hline \multirow{5}{*}{$\begin{array}{l}\text { Requisitos } \\
\text { logísticos }\end{array}$} & Treinamento & Reciclagem & $\begin{array}{l}\text { Na indústria, para qualquer } \\
\text { projeto vendido é necessário } \\
\text { que haja a previsão de } \\
\text { treinamento da equipe usuária } \\
\text { e de manutenção. Há sempre a } \\
\text { previsão de treinamentos } \\
\text { iniciais para apresentação do } \\
\text { novo projeto, como } \\
\text { treinamento de reciclagem } \\
\text { para eliminação de vícios de } \\
\text { uso. }\end{array}$ \\
\hline & $\begin{array}{l}\text { Assistência } \\
\text { Técnica }\end{array}$ & Presencial & $\begin{array}{l}\text { A parte de assistência técnica } \\
\text { é fundamental no pós-venda } \\
\text { de um projeto novo. A } \\
\text { assistência remota é usada } \\
\text { inicialmente para se entender a } \\
\text { dificuldade do detentor do } \\
\text { projeto. A dificuldade pode ser } \\
\text { de uso ou de manutenção. } \\
\text { Entendida esta dificuldade ela } \\
\text { pode ser resolvida por um } \\
\text { simples contato telefônico, de } \\
\text { vídeo ou até mesmo por } \\
\text { interferência remota de rede. } \\
\text { Caso não seja possível } \\
\text { resolver o problema por } \\
\text { nenhum dos meios } \\
\text { remotamente, aciona-se a } \\
\text { assistência técnica presencial. } \\
\text { A assistência técnica remota é } \\
\text { sempre a mais indicada devido } \\
\text { ao baixo custo desembolsado. }\end{array}$ \\
\hline & \multirow{2}{*}{ Manutenção } & Preditiva & $\begin{array}{l}\text { A manutenção de qualquer } \\
\text { equipamento mecânico se dá } \\
\text { de forma preditiva, quando há } \\
\text { o planejamento para a troca } \\
\text { periódica de peças e revisão do } \\
\text { funcionamento, ou então de } \\
\text { forma corretiva que pode }\end{array}$ \\
\hline & & Corretiva & $\begin{array}{l}\text { ocorrer por mau uso do } \\
\text { equipamento, falta de } \\
\text { manutenção preditiva ou então } \\
\text { por defeito de fabricação. } \\
\text { Ambas as manutenções devem } \\
\text { estar previstas durante a vida } \\
\text { útil do equipamento. }\end{array}$ \\
\hline & $\begin{array}{l}\text { Peças } \\
\text { sobressalentes }\end{array}$ & - & $\begin{array}{l}\text { As peças sobressalentes são } \\
\text { os materiais indispensáveis }\end{array}$ \\
\hline
\end{tabular}




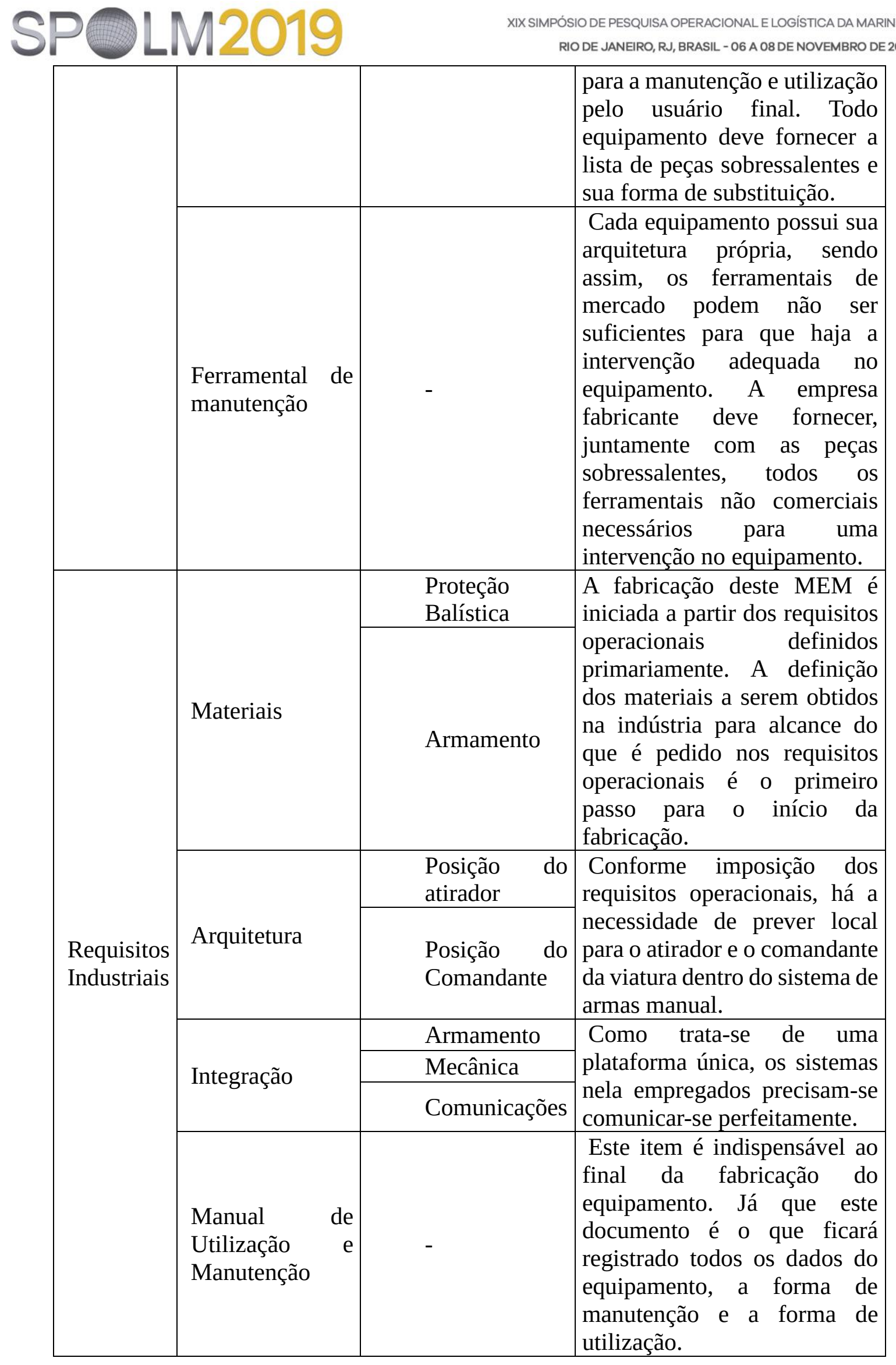

Os requisitos logísticos na sua forma tabular estão representados pela Tabela Requisitos Logísticos do Sistemas de Armas Manual. 
Tabela Requisitos Logísticos do Sistemas de Armas Manual

\section{ID REQUISITO \\ LOGÍSTICO}

DESCRIÇÃO

O AGR deve prover

Log01 Treinamento
Básico

Log02 $\begin{aligned} & \text { Treinamento } \\ & \text { Reciclagem }\end{aligned}$

Assistência

Log03 Técnica

Remota

Assistência

Log04 Técnica

Presencial

Log05 $\begin{aligned} & \text { Peças } \\ & \text { Sobressalentes }\end{aligned}$

fornecimento de

peças por no

mínimo 10 anos

O AGR deve

Log06 $\begin{aligned} & \text { Ferramental de } \\ & \text { Manutenção }\end{aligned}$

fornecer 1 kit

ferramental de

manutenção para

cada SAM

O AGR deve ser

Log07 $\begin{aligned} & \text { Manutenção } \\ & \text { Preditiva }\end{aligned}$

Log08 $\begin{aligned} & \text { Manutenção } \\ & \text { Corretiva }\end{aligned}$ manter um programa de manutenção preditiva do sistema

O AGR deve ser manter equipe pronta para realização de

$\begin{array}{lll}\text { REQUISITO } & \text { DERIVADO } & \text { CONTENÇÃO } \\ \text { DERIVADO } & \text { DE } & \text { REQUISITO } \\ \text { REQUISITO }\end{array}$

Log02

$\log 01$

$\log 01 \quad \log 01$

Log04

$\log 01$

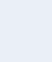
manutenção corretiva, quando acionado<smiles>O=S(=O)(O)O[Na]</smiles>

Log05; Log06

Da mesma forma, os requisitos industriais foram obtidos tendo como partida os requisitos operacionais definidos e são representados de forma tabular pela Tabela.Erro! Fonte de referência não encontrada.. 
TabelaErro! Fonte de referência não encontrada..

\section{ID $\begin{aligned} & \text { REQUISITO } \\ & \text { INDUSTRIAL DESCRIÇÃO }\end{aligned}$}

\section{REQUISITO DERIVADO CONTENÇÃO DERIVADO DE REQUISITO REQUISITO}

\begin{tabular}{|c|c|c|c|c|c|}
\hline Ind01 & $\begin{array}{l}\text { Materiais: } \\
\text { proteção } \\
\text { balística }\end{array}$ & $\begin{array}{l}\text { O AGR deve construir o } \\
\text { SAM com proteção } \\
\text { balística para } .50 \text { e } 7,62\end{array}$ & & & \\
\hline Ind02 & $\begin{array}{l}\text { Materiais: } \\
\text { armamento }\end{array}$ & $\begin{array}{l}\text { O AGR deve construir o } \\
\text { SAM com local para } \\
\text { armamento fixo }\end{array}$ & Ind01 & & \\
\hline Ind03 & $\begin{array}{l}\text { Arquitetura: } \\
\text { posição do } \\
\text { atirador }\end{array}$ & $\begin{array}{l}\text { O AGR deve desenhar o } \\
\text { o SAM de modo a } \\
\text { permitir um homem de } \\
\text { estatura média de } \\
180 \mathrm{~cm} \text { como atirador }\end{array}$ & Ind04 & & Ind04 \\
\hline Ind04 & $\begin{array}{l}\text { Arquitetura: } \\
\text { Posição do } \\
\text { Cmt }\end{array}$ & $\begin{array}{l}\text { O AGR deve desenhar o } \\
\text { SAM de modo a } \\
\text { permitir um homem de } \\
\text { estatura média de } \\
180 \mathrm{~cm} \text { como Cmt na } \\
\text { cabine com o atirador }\end{array}$ & & Ind03 & Ind03 \\
\hline Ind05 & $\begin{array}{l}\text { Integração: } \\
\text { Armamento }\end{array}$ & $\begin{array}{l}\text { O AGR deve realizar a } \\
\text { integração do } \\
\text { armamento com a } \\
\text { arquitetura do SAM }\end{array}$ & & & Ind06; Ind07 \\
\hline Ind06 & $\begin{array}{l}\text { Integração: } \\
\text { Mecânica }\end{array}$ & $\begin{array}{l}\text { O AGR deve realizar a } \\
\text { integração mecânica da } \\
\text { torre à plataforma e do } \\
\text { armamento ao SAM }\end{array}$ & & & Ind05; Ind07 \\
\hline Ind07 & $\begin{array}{l}\text { Integração: } \\
\text { Comunicações }\end{array}$ & $\begin{array}{l}\text { O AGR deve ser manter } \\
\text { um sistema de } \\
\text { comunicação entre o } \\
\text { atirador, Cmt e } \\
\text { motorista }\end{array}$ & & & Ind05; Ind06 \\
\hline Ind08 & $\begin{array}{l}\text { Incorporação } \\
\text { de periscópio }\end{array}$ & $\begin{array}{l}\text { O AGR deve ser } \\
\text { posicionar um } \\
\text { periscópio de visão } \\
\text { diurna para o atirador e } \\
\text { o Cmt }\end{array}$ & & & \\
\hline Ind09 & $\begin{array}{l}\text { Manual do } \\
\text { 9Usuário e de } \\
\text { Manutenções }\end{array}$ & $\begin{array}{l}\text { O AGR deve fornecer } \\
\text { o manual do usuário e } \\
\text { de manutenções para } \\
\text { cada SAM fabricado }\end{array}$ & & & Ind01 a Ind08 \\
\hline
\end{tabular}

A forma tabular é uma das formas de apresentação dos requisitos. Foi escolhida para confecção primeiramente devido à sua facilidade de visualização macro das sintaxes dos requisitos e a ligação entre eles. Porém, as representações dos requisitos também foram feitas através da forma padrão, através do diagrama de requisitos mostrados nas figuras abaixo. 


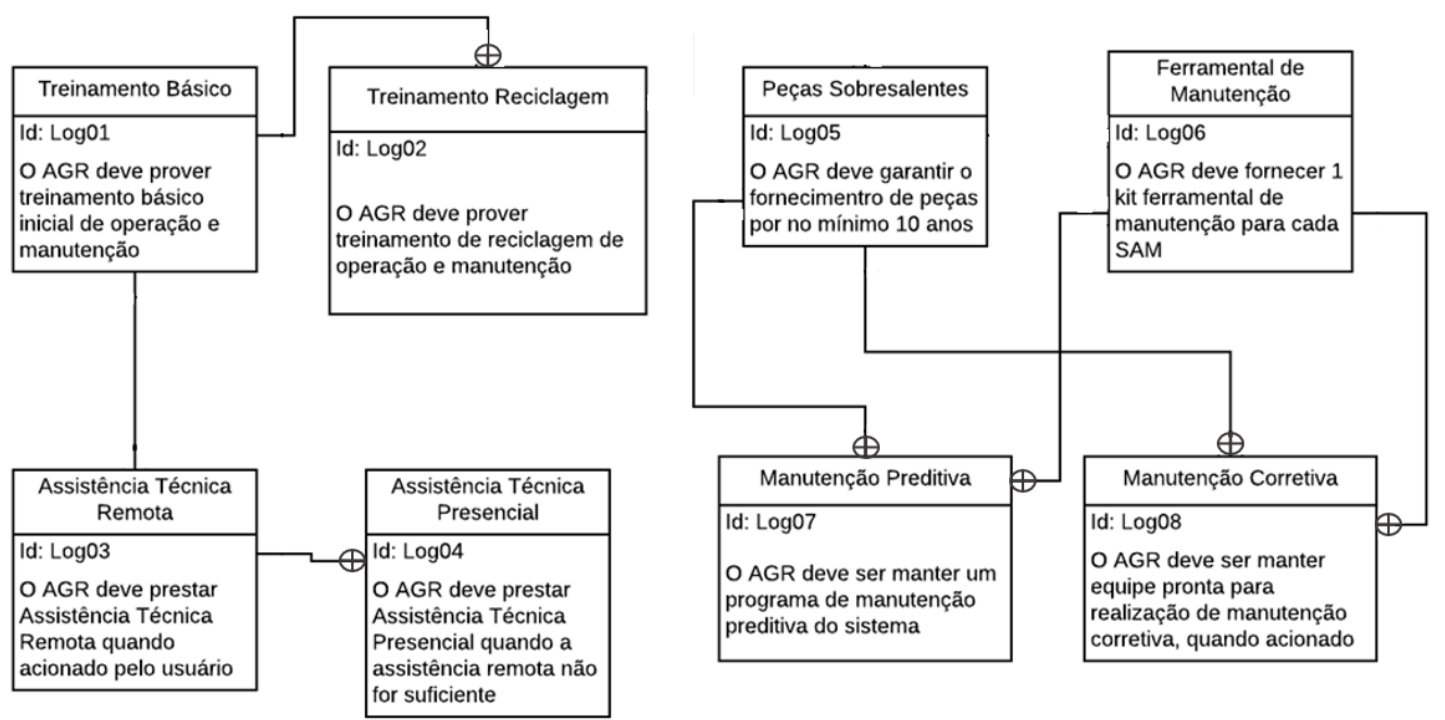

Diagrama de Requisitos Logísticos do Sistemas de Armas Manual

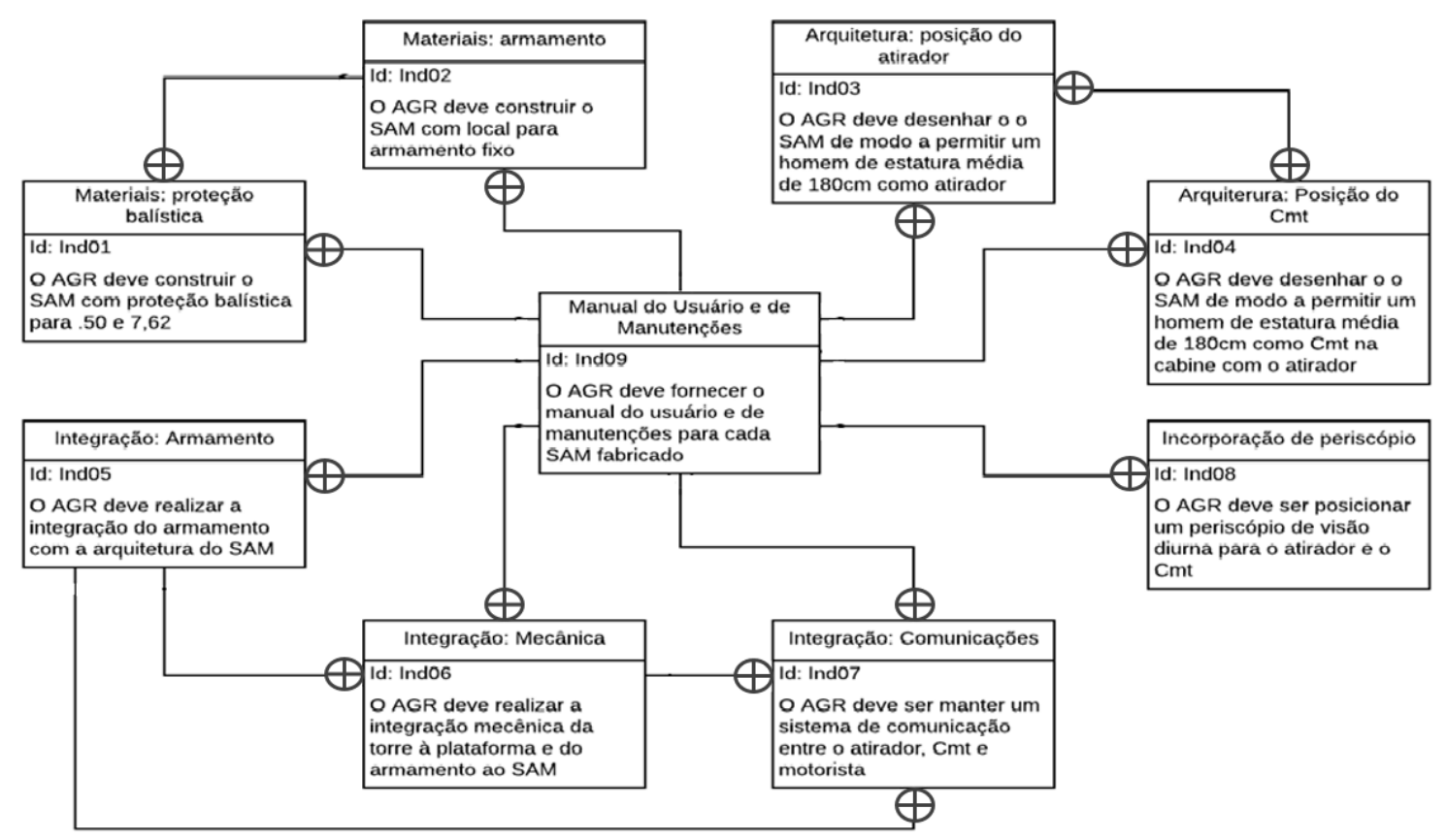

Diagrama de Requisitos Industriais do Sistemas de Armas Manual

\section{CONCLUSÃO}

A ESS se mostrou uma ferramenta extremamente apropriada e que já deveria estar em uso no âmbito do Exército Brasileiro, especialmente nos seus processos de aquisições de sistemas complexos, como no caso do Programa GUARANI. Seu estudo permitiu observar lacunas relevantes como a aquisição em separado de sistemas como os de Comando e Controle e o Sistema de Armas Manual; a aquisição de 03 (três) sistemas distintos de armas (automatizado 
e manual), o que pode ser considerado como um fator de dificuldade logística; a ausência de gestão de riscos, o que pode ser visto como uma dificuldade significativa na gestão do Programa pelo Gerente do Programa, uma vez que todo o sistema é afetado direta ou indiretamente como no caso da forte restrição orçamentária que ensejou severas alterações no escopo do Programa.

A visão sistêmica proporcionada pela ESS garante ao Gerente do Programa e aos Stakeholders uma possibilidade de alcançar sucesso superior a outras metodologias e precisa, salvo melhor juízo, ser introduzida nos programas de aquisições da Força, nos programas e projetos de desenvolvimento de PRODE, nos processos de aquisições, nos sistemas de fabricação e mesmo em sistemas de garantia da qualidade.

Este trabalho mostrou de forma preliminar como a ESS pode ser usada para a definição dos requisitos logísticos industriais para a definição de requisitos para SMEM. Apesar da abordagem ter sido de forma prospectiva, já é possível utilizar estes grandes temas para os trabalhos em andamento da Diretoria de Fabricação.

O processo de sete tarefas proposto pelo INCOSE, definido inicialmente, é uma excelente representação da engenharia de sistemas como é atualmente praticada e deve servir para evitar a maioria dos problemas que têm atormentado o desenvolvimento de sistemas grandes e complexos no passado.

Entenda todo o problema antes de tentar resolvê-lo;

Traduzir o problema em requisitos mensuráveis;

Examine todas as alternativas viáveis antes de selecionar uma solução;

> Certificar-se de considerar o ciclo de vida total do sistema. O conceito do nascimento até a morte se estende à manutenção, substituição e descomissionamento. Se isso não for considerado nas outras tarefas, os principais custos do ciclo de vida podem ser ignorados;

$>$ Certificar-se de testar o sistema total antes de entregá-lo;

$>$ Documente tudo.

No entanto, para avançar como uma disciplina e como uma profissão, a engenharia de sistemas deve crescer da minimização de problemas para a otimização do projeto pela integração dessas tarefas em uma teoria mais unificada. Elementos dessa teoria incluem o gerenciamento quantitativo de riscos, o projeto baseado em decisões e o gerenciamento de modelos matemáticos multidimensionais. À medida que o campo avança nessas e em direções similares, ganhará respeito adicional pela indústria, governo e academia. 


\section{REFERÊNCIAS BIBLIOGRÁFICAS}

[1] JUSTO, Andreia Silva. In: EAP(Estrutura Analítica do Projeto): o que é, como fazer

e qual a diferença entre EAP e Cronograma. 11 dez. 2018. Disponível em: https://www.euax.com.br/2018/12/eap-estrutura-analitica-projeto/. Acesso em: 22 ago. 2019.

[2] LEÃO, Thiago. In: NOMUS (Rio de Janeiro). Gráfico de Gantt: o que é, como funciona e como montar o seu. Rio de Janeiro: 13 jun. 2019. Disponível em: https://www.nomus.com.br/blog-industrial/grafico-de-gantt/. Acesso em: 19 ago. 2019.

[3] DIAGRAMA de bloco. 6 ago. 2016. Disponível em: https://www.conhecimentogeral.inf.br/diagrama_de_bloco/. Acesso em: 23 ago. 2019.

[4] GOVERNO EUA (Virginia). Defense Acquisition University. DEFENSE ACQUISITION GUIDEBOOK. 2. 25 set. 2017. Disponível em: https://www.dau.edu/. Acesso em: 12 ago. 2019.

[5] SMARTSHEET: Plataforma de execução de trabalhos baseada em nuvem. Versão 1.3.9. Bellevue, Washington: Mark Mader, 2005. Disponível em: https://app.smartsheet.com/new. Acesso em: 7 ago. 2019.

[6] LUCIDCHART: Todd McKinnon, Disponível em: https://www.lucidchart.com/pages/home?noHomepageRedirect=true. Acesso em: 7 ago. 2019.

[7] SIMPLEMIND. Disponível em: https://simplemind.eu/. Acesso em: 14 ago. 2019.

[8] INCOSE. SysML Modeling Language, 2006.

[9] SysML Open Source Project. SysML open source - Project index page. 